\title{
ORIGINAL
}

\section{SUPERVIVENCIA EN CÁNCER DE MAMA TRAS 10 AÑOS DE SEGUIMIENTO EN LAS PROVINCIAS DE GRANADA Y ALMERÍA}

Olga Ocón Hernández (1,2), Mariana F. Fernández Cabrera $(2,3,4)$, Sabina Pérez Vicente (5), Cristina Dávila Arias (6), José Expósito Hernández (7) y Nicolás Olea Serrano $(2,3,4)$

(1) UGC de Ginecología y Obstetricia. Hospital Universitario San Cecilio, Granada.

(2) Laboratorio de Investigaciones Médicas, Hospital Universitario San Cecilio, Granada.

(3) Centro de Investigación Biomédica, Universidad de Granada, Granada.

(4) CIBER en Epidemiología y Salud Pública (CIBERESP), España.

(5) Unidad de Apoyo a la Investigación. Empresa Pública Hospital Costa del Sol, Málaga.

(6) Servicio de Medicina Nuclear. Hospital Universitario San Cecilio, Granada.

(7) Servicio de Radioterapia. Hospital Universitario Virgen de las Nieves, Granada.

\section{RESUMEN}

Fundamento: Describir la supervivencia global y libre de enfermedad a los cinco y diez años del diagnóstico de cáncer de mama en mujeres participantes un estudio caso control previo, y establecer las variables pronóstico relacionadas.

Métodos: Se realizó el seguimiento de 202 mujeres diagnosticadas en tres hospitales públicos de Granada y Almería entre 19961998. La supervivencia se evaluó mediante el método de KaplanMeier, y la identificación de factores relacionados mediante el análisis de regresión de Cox.

Resultados: La edad media al diagnóstico fue de 54,27 $\pm 10,4$ años. La supervivencia global a los 5 años fue del $83,9 \%$ (IC $95 \%$ : $78,13-89,66$ ) y a los 10 años del 71\% (IC 95\%: 63,25-78,74) con un tiempo medio de seguimiento de 119,91 meses (IC 95\% 113,65126,17). La supervivencia libre de enfermedad a los 5 años fue del $81 \%$ (IC 95\%: 74,52-87,47) y a los 10 años del 71,3\% (IC 95\%: $63,33-79,26)$ con un tiempo medio de seguimiento de 118,75 meses (IC 95\% 111,86-125,65). La mortalidad de la serie fue del 33'17\%.

Conclusiones: Las características de la enfermedad en las mujeres de la muestra estudiada son similares a las de otras regiones de España y Europa, con una supervivencia global superior a la descrita en Europa y y comparable a la de España amba referidas para el mismo periodo.

Palabras clave: Cáncer de mama. Análisis de supervivencia. factores pronóstico.

\section{ABSTRACT \\ Breast Cancer Survival After 10 Years of Follow up, in Granada and Almeria Spanish Provinces}

Background: To describe the overall and disease-free survival at five and ten years after breast cancer diagnosis in women from a previous case-control study, and establish related prognostic factors.

Methods: We followed up 202 patients diagnosed between 1996 and 1998 in three public hospitals in Granada and Almeria provinces in Spain. Survival rates were calculated using the Kaplan and Meier method, and the Cox proportional hazards model was applied to identify the most significant variables contributing to survival.

Results: Mean age at diagnosis was $54.27 \pm 10.4$ years. Mean follow-up for overall survival was 119.91 months (95\%CI 113.65 $126.17)$; the five-year survival rate was $83.9 \%$ (95\% CI: 78.13-89.66) and the ten-year rate was $71 \%(95 \% \mathrm{CI}$ : $63.25-78.74)$. Mean followup for disease-free survival was 118.75 months (95\%CI 111.86 $125.65)$; the five-year disease-free survival rate was $81 \%(95 \% \mathrm{CI}$ : 74.52-87.47) and the ten-year rate was 71.3\% (95\%CI: 63.33-79.26). The mortality rate of the study population was $33.17 \%$.

Conclusions: Disease characteristics are similar in our population to those in other Spanish and European regions, while the overall survival is higher than the mean rate during the same period in Europe (5-yr rate of $79 \%$ ) and similar to that in Spain (83\%).

Key words: Breast cancer. Survival rates. Prognosis.

Financiación: Este trabajo ha sido financiado por proyectos de investigación de la Comisión Europea (7th. Frame Work, CONTAMED N ${ }^{\mathrm{o}}$ : 212502) y de la Consejería de Salud de la Junta de Andalucía (SAS-0133/2007). OOH y SPV disfrutan de contratos de investigación «Rio Hortega» y «Técnico de Apoyo», respectivamente, financiados por el Instituto de Salud Carlos III. Por su parte MFF es receptora de un contrato Ramón y Cajal financiado por el Ministerio de Ciencia e Innovación.
Correspondencia:

Mariana F. Fernández Cabrera

Laboratorio de Investigaciones Médicas.

Hospital Universitario San Cecilio

Granada

marieta@ugr.es 


\section{INTRODUCCIÓN}

El cáncer de mama es el tumor maligno de mayor incidencia y mortalidad entre las mujeres europeas, constituyendo un importante problema de salud pública en los países desarrollados. Programas como Surveillance, Epidemiology, and End Results (SEER) de Estados Unidos ${ }^{1}$ han analizado los cambios en los patrones de incidencia, mortalidad y supervivencia en cáncer, confirmando que, específicamente, el cáncer de mama es la causa más frecuente de muerte en mujeres entre 40 y 55 años, y que si la tendencia no cambia una de cada ocho mujeres desarrollará cáncer de mama a lo largo de su vida. El impacto sanitario, social y económico es, por tanto, realmente importante, teniendo en cuenta que afecta fundamentalmente a mujeres en edades tempranas y que en un tercio de los casos se convierte en una enfermedad crónica e insidiosa ${ }^{2}$.

Las diferencias regionales en incidencia en cáncer de mama otorgan un papel causal a las características geográficas, sociales y medioambientales de cada población ${ }^{3,4}$. Así, por ejemplo, España presenta una tasa de incidencia menor a la media de Europa, ocupando una posición intermedia entre los países occidentales y del este de Europa, aunque también se trata del tumor maligno más frecuente entre la población femenina ${ }^{3}$. Anualmente en nuestro país se diagnostican cerca de 16.000 casos nuevos, lo que supone casi el 30\% de los tumores descritos entre las mujeres. Según la Agencia Internacional de Investigación del Cáncer (IARC) ${ }^{3}$, la tasa de incidencia estandarizada estimada para España fue de 93,6 casos por 100.000 mujeres-año en 2006, frente a los 110 casos por cada 100.000 mujeres en Europa.

Aunque la información disponible en las diferentes regiones de España es insuficiente, los datos demuestran un incremento progresivo en la incidencia de esta patología en las dos últimas décadas (2-3\% anual) en todos los grupos de edad, confirmándose, además, como la primera causa de muerte por cáncer en las mujeres españolas (mortalidad estandarizada de 18,6 por 100.000 mujeres-año $)^{3,5,6}$. La supervivencia global a los 5 años era del 75\% entre los años 19901994, cercana a la referida en Europa que se situó en torno al $80 \%$, pero sensiblemente inferior a la descrita en Estados Unidos que alcanzó el $90 \%$ entre 1996 y $1998^{1,7,8}$. No obstante, las campañas de diagnóstico precoz y los avances terapéuticos han contribuido favorablemente al descenso paulatino en mortalidad de cerca del 2,4\% anual desde $1993^{3}$.

El cáncer de mama constituye un importante problema de salud también en Andalucía, donde es el tumor maligno más frecuente en las mujeres, con una incidencia en el año 2002 de 2.500 nuevos casos (67 casos por cada 100.000 mujeres $)^{9}$. Es, a su vez, la primera causa de muerte por cáncer en el colectivo femenino, con una tasa bruta de mortalidad de 24,1 por cada 100.000 habitantes en $1999^{9}$. Al igual que ocurre en otras regiones de España, se ha observado un descenso en la mortalidad a partir de 1993, lo que podría atribuirse, entre otras cosas, al mayor conocimiento de la enfermedad, a la mejora del tratamiento, y/o al diagnóstico en fases más tempranas, aunque no parece estar relacionado con el programa de detección precoz, ya que en Andalucía se inició a mediados de los años noventa ${ }^{10}$ y es pronto para ver sus efectos sobre mortalidad.

Mientras que existe un consenso a la hora de establecer los principales factores de riesgo para esta patología (características reproductivas -menarquia, menopausia, paridad-, obesidad, estatus socioeconómico, susceptibilidad genética, y de manera menos consistente, dieta) ${ }^{3}$, los factores pronósticos y predictivos se han ido definiendo a lo largo de los últimos años, paralelamente a los avances en las ciencias básicas, el desarrollo de nuevas técnicas de investigación y el conocimiento de los procesos moleculares implicados en la patogenia de la enferme- 
dad $^{11}$. Así, se admiten como factores pronósticos tanto las características de la enfermedad (tamaño tumoral, tipo y grado histológico e invasión vascular y estado de los ganglios axilares) y de las mujeres afectadas ${ }^{12}$ (edad, estilo de vida, características reproductivas), como algunos biomarcadores (receptores de hormonas esteroideas RE$\mathrm{RP}$, sobreexpresión de c-erbB-2, p53), aunque algunos de ellos parecen no tener un papel claramente consensuado, especialmente a la hora de establecer su influencia en la supervivencia ${ }^{13}$.

Con objeto de investigar los factores de riesgo en cáncer de mama se diseñó un estudio caso-control, reclutando entre 19961998 a 202 mujeres recién diagnosticadas de esta patología en tres hospitales públicos de las provincias de Granada y Almería ${ }^{13}$. Las estimaciones de riesgo de cáncer de mama fueron estadísticamente significativas para las variables estado civil, nivel educativo, ocupación y clase económico-social, así como número de embarazos, paridad, meses acumulados de lactancia, antecedentes familiares de cáncer de mama y consumo de alcohol y tabaco. Transcurridos diez años desde el reclutamiento de las mujeres, se planteó conocer su estado vital y estudiar la influencia de las variables epidemiológicas de riesgo.

El objetivo de este trabajo es describir la supervivencia global y libre de enfermedad a los cinco y diez años en este grupo de mujeres y definir las variables pronóstico relacionadas con su supervivencia.

\section{SUJETOS Y MÉTODOS}

Entre 1996-1998 en los hospitales Universitario San Cecilio y Virgen de las Nieves de Granada, y Torrecárdenas de Almería, 202 mujeres diagnosticadas e intervenidas por cáncer de mama que cumplían los criterios de inclusión establecidos, fueron apareadas por edad y hospital con otras 260 muje- res intervenidas de patología no oncológica, ni ginecológica ni endocrina. Toda la información del estudio caso-control de cáncer de mama se detalla en el trabajo original de Ibarlucea y cols. $2004^{13}$. El rango de edad de las participantes fue de 35 a 76 años. Las fuentes de información del estudio fueron la historia clínica, un cuestionario epidemiológico personal estructurado y diseñado $a d$ hoc., y muestras biológicas obtenidas durante la cirugía. Transcurridos diez años desde el inicio del estudio se ha recogido la información sobre la evolución clínica de la enfermedad y el estado vital de las pacientes, cotejándolo con los datos disponibles en el Registro del Cáncer de Granada y en el Registro Nacional de Defunciones. Se han seguido los protocolos establecidos por los centros sanitarios implicados para acceder a los datos de las historias clínicas.

En base a la información obtenida en el trabajo inicial y la revisión del estado vital de las mujeress a los 10 años, las variables supervivencia global y libre de enfermedad a los cinco y diez años se construyeron en función del tiempo transcurrido desde el momento del diagnóstico hasta la ocurrencia de un evento (recidiva, metástasis o éxitus) o fecha de último contacto, estableciendo como límite de seguimiento el mes de abril de 2008. Las variables recogidas en la revisión de las historias clínicas fueron: estadío clínico y postquirúrgico, tipo y grado histológico, recidiva y metástasis (tiempo y localización) y fecha de éxitus. Se siguieron los criterios del American Join Commission on Cancer (AJCC) ${ }^{14}$ para la clasificación del estadio y se definió como recaída de la enfermedad la presentación de recidiva local o de metástasis a distancia.

Análisis estadístico. En el análisis descriptivo se incluye el valor de la mediana, media, rango y desviación estándar para las variables cuantitativas y las medidas de frecuencia en el caso de variables categóricas. La estimación de la función de supervivencia se ha realizado mediante el método de 
Kaplan-Meier, calculando la supervivencia global y la supervivencia libre de enfermedad para cada una de las características de la enfermedad. Para el análisis de aquellos factores que potencialmente podrían modificar la supervivencia se ha empleado la regresión de Cox. Se consideró un valor de $\mathrm{p}<0,05$ como diferencia estadísticamente significativa. Para la creación de la base de datos y el análisis estadístico se utilizó el paquete estadístico SPSS 15.0.

\section{RESULTADOS}

La edad media de las mujeres en el momento del diagnóstico fue de 54,27 años (tabla 1). Los 202 casos de cáncer de mama incluidos en el estudio se clasificaron atendiendo a las características de la enfermedad (tipo y grado histológico y localización de las metástasis) (tabla 2). El 74,25\% de los casos correspondieron a carcinoma ductal, incluyendo 3 casos de carcinoma ductal in situ $(1,48 \%)$. El porcentaje de carcinoma lobulillar fue del $6,93 \%$ y el $11,40 \%$ restante correspondió a otros tipos histológicos. El grado histológico más frecuentemente descrito entre las pacientes fue el $3(47,1 \%)$, seguido del $2(41,29 \%)$ y, por último, el grado $1(10,32 \%)$ de los casos. Se demostró invasión ganglionar en 84 (41,58\%) mujeres.

La tabla 3 muestra la distribución de los casos por estadío antes de la cirugía o estadío clínico, atendiendo a los criterios establecidos por la AJCC. Las mujeres se volvieron a clasificar tras la cirugía, lo que permitió establecer la concordancia entre la clasificación pre- y post-quirúrgica. En el diagnóstico de estadios más precoces (IA y IIA) la concordancia fue del 71,9\% y para estadios avanzados (IIIA y IIIB) del 84,3\%, mucho mayor respecto a los estadíos intermedios cuya concordancia entre el diagnóstico pre y postquirúrgico fue del $18,3 \%$.

El tiempo medio de seguimiento de las 202 mujeres incluidas fue de 92,32 $\pm 33,38$ meses, con un rango de 6 a 135 meses. $\mathrm{Al}$ final de dicho periodo se habían producido un total de 67 defunciones $(33,17 \%)$ con una edad media a la defunción de 60,33 $\pm 11,632$ años, con 37 años la más joven y 82 la de mayor edad. A lo largo del seguimiento 58 $(28,71 \%)$ mujeres presentaron algún tipo de recaída, evidenciándose recidivas en 18 $(8,91 \%)$, tanto locales, contralaterales, como en la piel de la mama afectada, así como 36 $(17,82 \%)$ metástasis, cuya localización se detalla en la tabla 4.

El análisis de supervivencia en las 186 $(92,08 \%)$ mujeres para las que se dispuso de información completa al final del seguimiento, muestra una supervivencia global a los 5 años del 83,9\% (IC 95\% 78,13-89,66) y a los 10 años del 71\% (IC 95\% 63,25-78,74), con un tiempo medio de supervivencia de 119,91 meses (IC 95\% 113,65-126,17)

Tabla 1

Edad al diagnóstico en cáncer de mama

\begin{tabular}{|l|r|r|}
\hline \multicolumn{1}{|c|}{ Edad (años) } & $\mathbf{n}$ & \% \\
\hline$<45$ & 42 & 20,79 \\
$45-54$ & 55 & 27,23 \\
$55-64$ & 53 & 26,24 \\
$>64$ & 36 & 17,82 \\
No disponible & 16 & 7,92 \\
Total & 202 & 100 \\
\hline
\end{tabular}


Tabla 2

Características de la enfermedad en las 202 mujeres diagnosticadas de cáncer de mama

\begin{tabular}{|l|r|r|}
\hline & $\mathbf{n}$ & $\mathbf{\%}$ \\
\hline Variedades histológicas (n=202) & & \\
- Carcinoma In situ & 3 & 1,48 \\
- Carcinoma infiltrante Ductal & & \\
- Forma clásica & 147 & 72,77 \\
- Comédoco & 6 & 2,97 \\
- Inflamatorio & 3 & 1,48 \\
- Inflamatorio+comédoco & 1 & 0,49 \\
- Medular & 2 & 0,99 \\
- Mucinoso/mucoide & 6 & 2,97 \\
- Papilar & 2 & 0,99 \\
- Epidermoide & 1 & 0,49 \\
- Paget+ductal infiltrante & 1 & 0,49 \\
- Carcinoma infiltrante Lobulillar & & \\
- Forma clásica & 14 & 6,93 \\
- Ambos (Ductal y Lobulillar) & 1 & 0,49 \\
- No disponible & 15 & 7,43 \\
\hline Grado histológico (n=202) & & \\
- Grado 0 & 1 & 0,5 \\
- Grado 1 & 16 & 7,8 \\
- Grado 2 & 65 & 31,9 \\
- Grado 3 & 74 & 36,3 \\
- Grado 4 & 1 & 0,5 \\
- No disponible & 47 & \\
\hline Localización metástasis (n=36) & & \\
- Pulmonares & 5 & 13,88 \\
- Óseas & 7 & 19,44 \\
- Hepáticas & 5 & 13,88 \\
- Cerebrales & 1 & 2,77 \\
- Supraclaviculares & 5,55 \\
- Cuero cabelludo & 2,77 \\
- Múltiples & & \\
\hline
\end{tabular}

(figura 1). El análisis de la supervivencia libre de enfermedad se realizó con datos disponibles para 174 mujeres $(86,14 \%)$, observándose una supervivencia libre de enfermedad a los cinco años del 81\% (IC 95\% 74,5287,47 ) y del $71 \%$ a los 10 años (IC 95\% $63,33-79,26)$, con un tiempo medio libre de enfermedad de 118,75 meses (IC 95\% 111,86-125,65) (figura 2).

En el grupo de mujeres que sufrieron algún tipo de recaída de la enfermedad (recidiva o metástasis), el tiempo medio libre de enfermedad fue de 51,18 $\pm 33,34$ meses, con 
Tabla 3

Distribución por estadio en cáncer de mama

\begin{tabular}{|c|c|c|c|}
\hline Estadio Clínico & $\mathbf{n}(\mathbf{\%})$ & $\begin{array}{c}\text { Estadio } \\
\text { Postquirúrgico }\end{array}$ & $\mathbf{n}(\mathbf{\%})$ \\
\hline I & $425(12,38)$ & I & $30(14,85)$ \\
IIA & $99(49,01)$ & IIA & $64(31,68)$ \\
IIB & $31(15,35)$ & IIB & $50(24,75)$ \\
IIIA & $12(5,94)$ & IIIA & $22(10,09)$ \\
IIIB & $6(2,97)$ & IIIB & $11(5,44)$ \\
IIIC & 0 & IIIC & $5(2,47)$ \\
IV & $4(1,98)$ & IV & $2(0,99)$ \\
No disponible & $25(12,37)$ & No disponible & $18(8,91)$ \\
Total & $202(100)$ & Total & $202(100)$ \\
\hline
\end{tabular}

Figura 1

Supervivencia global

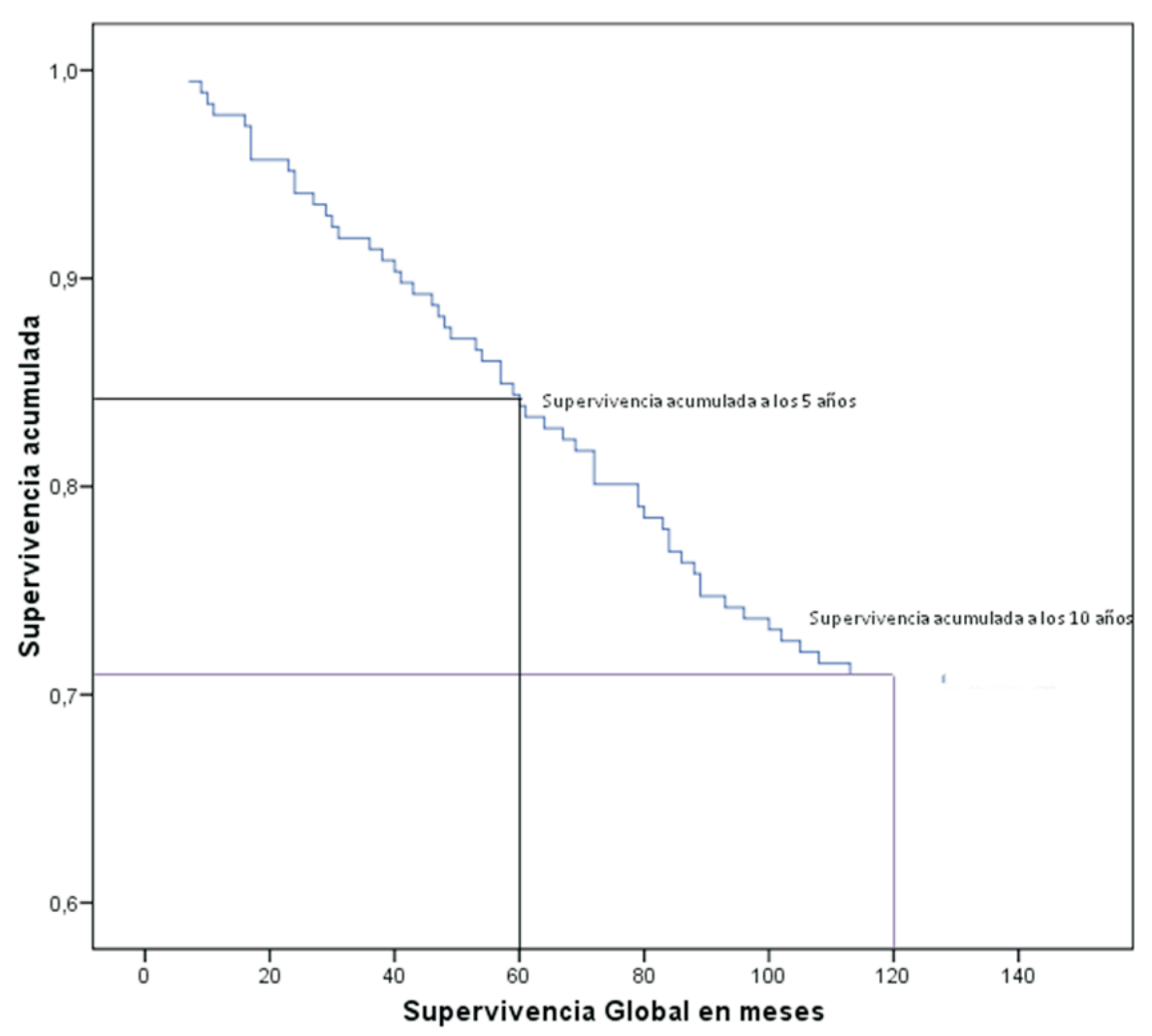


Figura 2

Supervivencia libre de enfermedad

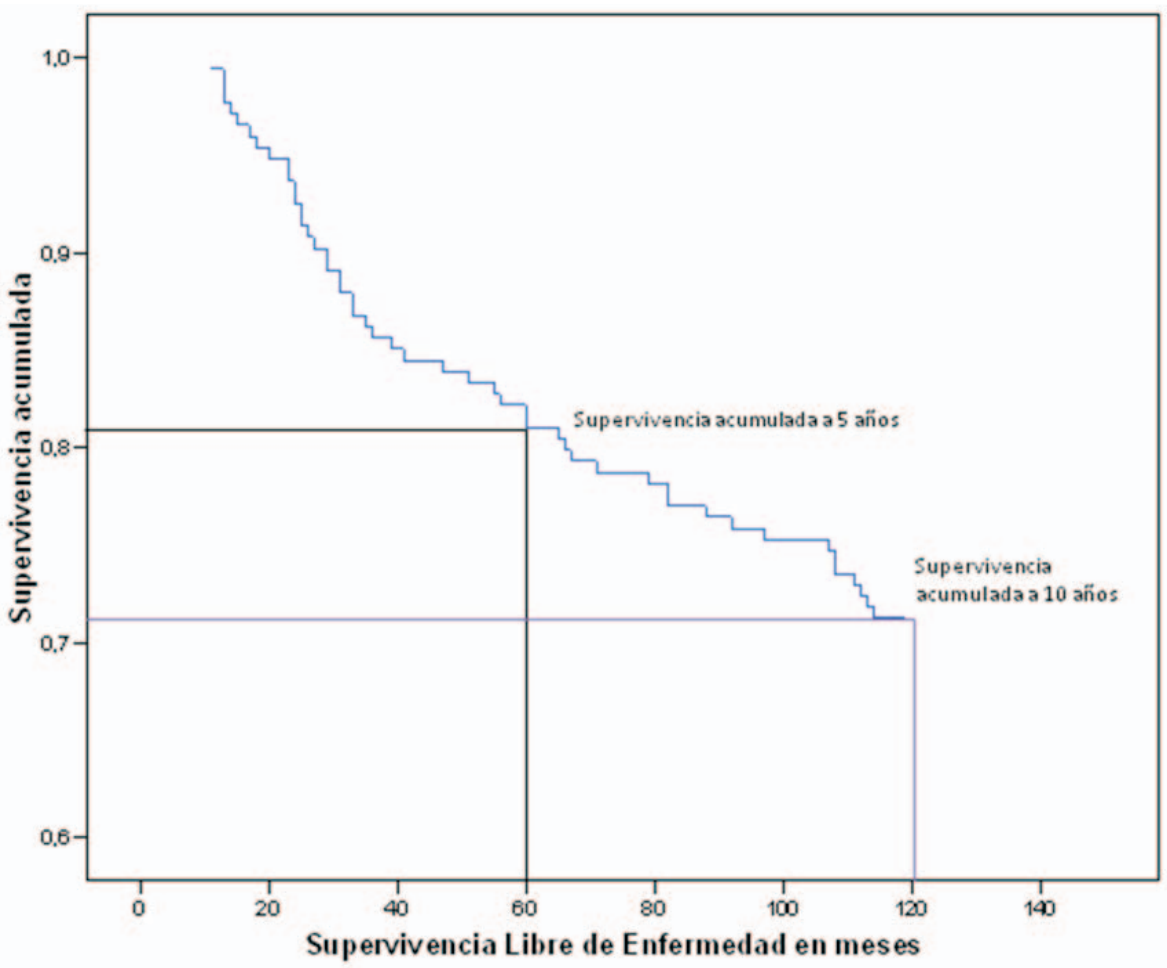

una mediana de 37,50 meses, un percentil $25 \%$ de 24 meses y un percentil $75 \%$ de 79,75 meses. Para los casos que no presentaron recidiva o metástasis, el tiempo de supervivencia medio fue de $126,20 \pm 23,51$

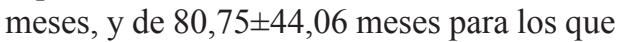
presentaron recaída de la enfermedad, siendo esta diferencia estadísticamente significativa $(\mathrm{p}<0,001)$. La supervivencia global de los casos que no presentaron metástasis a lo largo del seguimiento fue del $90,1 \%$, frente al $19,5 \%$ de los que sí la presentaron $(\mathrm{p}<0,001)$.

En el estudio de la relación entre supervivencia global y libre de enfermedad y la edad de las mujeres en el momento del diagnóstico así como con las características de la enfermedad, no se encontró ninguna asocia- ción estadísticamente significativa entre supervivencia y edad, independientemente de la utilización de esta variable de manera continua o categórica. Tampoco se ha podido establecer ninguna asociación estadísticamente significativa entre la edad y características de la enfermedad, tales como estadio, grado y tipo histológico.

La supervivencia global se estudió atendiendo a los tipos histológicos previamente descritos, de manera que para los carcinomas ductales, con sus variantes celulares, la supervivencia fue del 83,2 y 70,3\% a los 5 y 10 años de seguimiento, respectivamente, con un tiempo medio de supervivencia de 119,3 meses (IC 95\% 112,42-126,17). Para los carcinomas lobulillares, la supervivencia fue del $85,7 \%$ a los 5 años y del $64,3 \%$ a los 
10 años, con un tiempo medio de supervivencia de 117,14 meses (IC 95\% 95,48$138,81)$. No se encontraron diferencias estadísticamente significativas en cuanto a supervivencia entre ambos tipos histológicos.

Entre los $171(84,65 \%)$ casos para los que se pudo conocer el estadio postquirúrgico, presentaron una mayor supervivencia global aquellos diagnosticados en los estadios menos avanzados, con una supervivencia global del $85,7 \%$ y del $71,4 \%$ a los 5 y 10 años para el estadio I y del $86,2 \%$ a los 5 años y del 77,6\% a los 10 años para el estadio II. El tiempo medio de supervivencia para los casos clasificados dentro del estadio I fue de 118,54 meses (IC 95\% 101,27135,8 ) y de 124,97 meses (IC 95\% 113,93$134,21)$ para el IIA. No se encontraron diferencias estadísticamente significativas entre supervivencia global y estadio postquirúrgico; sin embargo, si se encontró asociación significativa $(p=0,014)$ entre la supervivencia libre de enfermedad y el estadio, aunque únicamente entre el estadio I y el IIA con una mayor supervivencia libre de enfermedad para el estadio I.

En nuestra serie, los casos clasificados en el grado 1 de diferenciación presentaron una supervivencia global del $93,8 \%$, tanto a los 5 como a los 10 años; con un tiempo medio de supervivencia de 136,87 meses (IC 95\% 127,15-146,6). La supervivencia descendió progresivamente a medida que aumentó el grado, llegando al $68,5 \%$ y $50,7 \%$ a los 5 y 10 años, respectivamente, para los casos con grado 3; con un tiempo medio de supervivencia de 99,93 meses (IC 95\% 88,22111,64). Las diferencias fueron estadísticamente significativas cuando se compararon todos los grados histológicos entre sí $(\mathrm{p}<0,001)$.

Por último, la invasión de los ganglios axilares determinó una supervivencia significativamente menor $(\mathrm{p}<0,001)$, con una supervivencia global a los 10 años del 57,6\% si había invasión y del $82,8 \%$ si no existía. La infiltración ganglionar también determinó una menor supervivencia libre de enfermedad $(p=0,047)$. La supervivencia libre de enfermedad a los 10 años para los casos sin invasión fue del 78,1\% y del 64,5\% si había metástasis ganglionar.

\section{DISCUSIÓN}

Las mujeres incluidas en nuestro estudio presentan características similares en supervivencia a las descritas previamente en Europa. Así, la supervivencia global tanto a los 5 años $(83,9 \%)$ como a los 10 años (71\%), son comparables a las descritas en el estudio EUROCARE, que estimó una supervivencia media para cáncer de mama a los 5 años del diagnóstico del 76,1\% entre los años 1983-1994 ${ }^{15}$, pero que ascendió al $82,2 \%$ para el periodo $1994-2003^{7}$, rango de años en el que se enmarca nuestra serie. Nuestros resultados también son comparables a los establecidos por la base de datos GLOBOCAN $2000^{8,16}$, que incluye los referidos en EUROCARE y SEER, y que considera una supervivencia media en Estados Unidos del $81 \%$ a los 5 años.

En España en particular, el estudio El Álamo sobre cáncer de mama llevado a cabo entre los años 1990-199717,18, refiere un tiempo medio de supervivencia global de 8,68 años/104,16 meses (IC95\%: 8,50/96,6$8,85 / 106,2)$, ligeramente inferior a los 9,99 años/119,91 meses (IC95\%: 113,65-126,17) descritos en nuestro estudio. Es interesante recordar a este respecto, que entre los 32 hospitales incluidos en el estudio de referencia no se encuentran las provincias de Granada y Almería, por lo que la información aquí recogida bien podría ayudar a componer el mapa de supervivencia en España.

En general, la supervivencia en mujeres con cáncer de mama en España ha mejorado ostensiblemente en las últimas décadas, 
pasando de un 64\% a los 5 años del diagnostico entre 1980-1985, a un 78\% en el periodo $1990-1994^{7,15}$. Pero además, si comparamos los resultados de supervivencia obtenidos en nuestra serie con dos estudios anteriores realizados en el mismo área geográfica en los años 80 y 90, es posible identificar una mejora en la precocidad del diagnóstico y en la supervivencia en sí misma. En el primero de los trabajos locales referidos ${ }^{19}$ se describe una edad media al diagnóstico de 56 años, similar a la de nuestro estudio, pero una distribución diferente en cuanto a subtipos histológicos y estadío. Así, la mitad de los tumores fueron carcinoma ductal infiltrante y en un $50 \%$ de los mismos el diagnóstico se efectuó en fases de la enfermedad localmente avanzada y/o distal, presentando un $49 \%$ de casos ganglios axilares positivos. La supervivencia global descrita a los 3 años de seguimiento fue del $82 \%$ y la supervivencia libre de enfermedad del $63 \%$. Por otra parte, el segundo de los trabajos ${ }^{20}$, publicado en 1996, incluye datos de un seguimiento más prolongado observando una supervivencia global a los 10 años del $60 \%$ y una supervivencia libre de enfermedad del $22 \%$.

El principal factor en la mejora de supervivencia en cáncer de mama, según la literatura, es la mejora en los tratamientos adyuvantes a la cirugía ${ }^{21}$. Por otro lado, el control de la enfermedad podría deberse también al efecto del cribado del cáncer de mama mediante mamografía, que permite diagnosticar estadíos más precoces de la enfermedad, aunque su eficacia sigue siendo controvertida $^{22}$. En España se instauró escalonadamente a partir de $1990^{23}$, aunque en Andalucía según datos del estudio DESCRIC, el programa no se inició hasta 1995, siendo su implantación incompleta e irregu$\operatorname{lar}^{24}$. No obstante el peso relativo de cada uno de estos factores está aún por diluci$\operatorname{dar}^{21}$.

Es cierto que la comparación de datos de supervivencia entre diferentes series se encuentra con dificultades derivadas tanto de la forma de presentación de los resultados, por ejemplo, el uso de la supervivencia relativa ${ }^{16}$, como por las características mismas de la población de estudio que pueden variar fuertemente en rangos de edad y estadios diagnósticos. Afortunadamente, las características generales de nuestra población se asemejan a las referidas en otros estudios realizados en España durante las dos últimas décadas, lo que permite la comparación de resultados. Así, el proyecto El Álamo describe una edad media al diagnóstico de 56,72 años, lo que se corresponde con los 54,27 años descritos en nuestra serie. De igual manera, en el estudio El Álamo, aproximadamente un tercio de los casos son diagnosticados en mujeres menores de 50 años, porcentaje similar a los datos observados en nuestra serie $(35,15 \%)$. Las características histológicas en nuestra serie también son equivalentes a las descritas en otros estudios, con una mayor presencia del tipo histológico ductal ${ }^{25}$, por ejemplo, el $81,7 \%$ de la población de El Álamo presentó carcinoma ductal frente al $74,25 \%$ en nuestra población, mientras que el tipo lobulillar supuso aproximadamente el $7 \%$ en ambos estudios.

Otra información de interés es la distribución por estadio, ya que constituye un indicador de la calidad del diagnóstico en la enfermedad. Cerca del $20 \%$ de las pacientes en el estudio El Álamo presentó tumores localmente avanzados (IIIA y IIIB). En nuestro estudio fueron diagnosticados en estadios avanzados sólo el 13,4\% de los casos. Estas cifras parecen excesivas para un país europeo, sin embargo podrían ser consecuencia, según los autores de El Álamo, del desconocimiento o miedo de la población femenina española frente a esta patología, lo que indica la necesidad de llevar a cabo, entre otras cosas, campañas de divulgación e información sobre esta enfermedad. 
El 48\% de las mujeres en el estudio El Álamo no tenía afectación de los ganglios axilares, porcentaje algo menor al descrito en nuestra población de estudio, con un 58\% de los casos con ganglios negativos. Esta característica de nuestra serie podría constituir un dato diferenciador, ya que la no afectación ganglionar es determinante para la supervivencia de las pacientes ${ }^{26}$ y justificar que la supervivencia global de nuestro estudio sea ligeramente superior a la descrita en El Álamo, situada en 119,91 meses frente a 104,16 meses de nuestra serie.

En la mayoría de los trabajos revisados el grado histológico más frecuente es el grado 2 , constituyendo aproximadamente el $60 \%$ de los casos diagnosticados ${ }^{17}$. Sin embargo, nuestra población presentó una distribución equivalente entre los grados 2 y 3, 41,29\% y $47,1 \%$, respectivamente. Un mayor grado histológico se ha asociado consistentemente con una disminución de la supervivencia a largo plazo $^{26}$, hecho que se confirma en nuestra serie donde la supervivencia a 5 y 10 años en las pacientes con grado 1 de diferenciación fue del 93,8\%, y para el grado 3 , del $68,5 \%$ a 5 años y $50,7 \%$ a 10 años.

Son muchos los factores pronósticos identificados en el seguimiento de las pacientes con cáncer de mama, aunque no todos han sido validados y reconocidos como tales en las diferentes series de pacientes ${ }^{11,25,26}$. Nuestro trabajo confirma el valor pronóstico de algunos de ellos (estadio al diagnóstico, que incluye la afectación de ganglios axilares y presencia de metástasis, y el grado histológico) y fracasa a la hora de poner de manifiesto la significación de otros. Las limitaciones inherentes a las características de nuestra muestra (cohorte de base hospitalaria con criterios de selección definidos a priori y con un reducido tamaño, junto con la validez de calidad de los registros utilizados, que por ejemplo impide evaluar de forma estratificada la edad), deben ser tenidas en cuenta a la hora de extraer conclusiones de orden general.

\section{BIBLIOGRAFÍA}

1. Surveillance, Epidemiology, and End Results (SEER) Program. SEER Cancer Statistics Review. 1975-2006. [Citado 29 Mayo, 2009]. Disponible en: http://seer.cancer.gov/.

2. Martín M, Mahillo E, Llombart-Cussac A, Lluch A, Munarriz B, Pastor M, et al. The «El Álamo» Project (1990-1997): two consecutive hospital-based estudies of breast cancer outcomes in Spain. Clin Transl Oncol. 2006; 8(7): 508-18.

3. Pollán M, Ramis R, Aragonés N, Pérez-Gómez B, Gómez D, Lope V et al. Municipal distribution of breast cancer mortality among women in Spain. BMC Cancer. 2007; 7:78.

4. Ruíz M, Viciana F. Tendencia y distribución geográfica de la mortalidad por cáncer de mama en Andalucía (1976-1995). Aten Primaria. 1997; 299304.

5. López-Abente G, Pollán M, Aragonés B, Pérez Gómez V, Hernández Barrera V, Lope V, et al. Situación del cáncer en España: incidencia. An Sist Sanit Navar. 2004; 27 (2): 165-73.

6. Díez MC, Ortega P, Villanueva R, Albaladejo R, Astasio P, Calle ME. Características epidemiológicas relacionadas con el cáncer de mama en mujeres pre y postmenopáusicas. Med Clin (Barc). 2000; 115: $281-6$.

7. Verdecchia A, Francisci S, Brenner H, Gatta G, Micheli A, Mangone L, et al. Recent cancer survival in Europe: a 2000-02 period analysis of EUROCARE-4 data. Lancer Oncol. 2007; 8 (9): 784-96.

8. Parkin DM, Bray F, Ferlay J, Pisani P. Global cancer statistics, 2002. CA Caner J Clin. 2005; 55 (2): 74-108.

9. Consejería De Salud. Plan Integral de Oncología de Andalucía 2002-2006. Disponible en www.juntadeandalucia.es.

10. Ruiz Ramos M, Expósito J. Tendencia de la mortalidad por cáncer en Andalucía entre 1975 y 2003. Med Clin (Barc). 2007;128(12): 448-52.

11. Domínguez MA, Marcos M, Meiriño R, Villafranca E, Dueñas MT, Arias F, et al. Factores pronósticos y predictivos en el cáncer de mama temprano. An Sist Sanit Navar. 2001; 24 (Supl 1): 99-110.

12. Patterson RE, Cadmus LA, Emond JA, Pierce JP. Physical activity, diet, adiposity and female breast 
cancer prognosis: A review of the epidemiologic literature. Maturitas. 2010; 66(1):5-15.

13. Ibarluzea JM, Fernandez MF, Santa Marina L, Olea MF, Rivas AM, Aurrekoetxea JJ, et al. Breast cancer risk and the combined effect of environmental estrogens. Cancer Causes Control 2004; 15:591600 .

14. Greene FL, Page DL, Fleming ID, et al, editors. AJCC Cancer staging manual, $6^{\text {th }}$ edition. New York: Springer; 2002.

15. Sant M, Aareleid T, Berrino F, Bielska Lasota M, Carli PM, et al. EUROCARE-3: survival of cancer patients diagnosed 1990-94, results and commentary. Ann Oncol 2003; 14 (Supl 5):61-118.

16. Parkin DM, Bray F, Ferlay J, Pisani P. Estimating the world cancer burden: GLOBOCAN 2000. Int J Cancer. 2001; 94: 153-6.

17. Martín M, Llombart-Cussac A, Lluch A, Alba E, Munarriz B, Tusquets I, et al. Epidemiologicyal study of the GEICAM group about breast cancer in Spain (1990-1993): El Alamo project. Med Clin (Barc). 2004;122: 12-7.

18. Martín M, Mahillo E, Llombart-Cussac A, Lluch A, Munarriz B, Pastor M, et al. The «El Alamo» project (1990-1997): two consecutive hospital-based studies of breast cancer outcomes in Spain. Clin Transl Oncol. 2006; 8 (7): 508-18.

19. Moral R del, Ruíz de Almodóvar JM, Villalba J, López Ruíz JM, Expósito J, García Puche JL, et al. Supervivencia global y supervivencia libre de enfermedad en cáncer de mama. Factores de influencia. Oncología (Barc). 1989; 12(2): 83-92.

20. Pérez Cabrera B. Factores pronósticos en el cáncer de mama y evaluación de los receptores hormona- les: estudio sobre 241 casos [Tesis Doctoral]. Granada: Universidad de Granada; 1996.

21. Rosso S, Gondos A, Zanetti R, Bray F, Zakelj M, Zagar T, et al; the EUNICE Survival Working Group. Up-to-date estimates of breast cancer survival for the years 2000-2004 in 11 European countries: The role of screening and a comparison with data from the United States. Eur J Cancer. En prensa.

22. Mc Tiernan A. Recente controversies in mammography screening for breast cancer. Medscape Womens Health 2002; 7(2):3.

23. Izquierdo A, Gispert R, Saladie F, Espinás JA. Análisis de la incidencia, la supervivencia y la mortalidad según las principales localizaciones tumorales, 1985-2019: cáncer de mama. Med Clin (Barc). 2008; 131 (Supl 1): 50-2.

24. Castells X, Sala M, Ascunce N, Salas D, Zubizarreta R, Casamitjana M, coordinadores. Descripción del cribado del cáncer en España. Proyecto DESCRIC. Madrid: Plan de Calidad para el Sistema Nacional de Salud. Ministerio de Sanidad y Consumo. Agència d'Avaluació de Tecnologia i Recerca Mèdiques de Cataluña; 2007. Informes de Evaluación de Tecnologías Sanitarias, AATRM núm. 2006/0.

25. Soerjomataram I, Louwman MWJ, Ribot JG, Roukema JA, Coebergh JWW. An overview of prognostic factors for long-term survivors of breast cancer. Breast Cancer Res Treat. 2008; 107: 309-30.

26. Flores-Luna L, Salazar-Martínez E, Duarte-Torres RM, Torres-Mejía G, Alonso-Ruíz P, Lazcano-Ponce E. Factores pronósticos relacionados con la supervivencia del cáncer de mama. Salud Publica Mex. 2008; 50:119-25. 